\title{
The use of the ICAM Splint Programme in Zone IV to VII Extensor Tendon Repairs: Patient outcomes and clinician experiences in a specialised hand unit in SA
}

\author{
*Nicole Mottay, B.OT (UKZN); M.OT (UKZN). https://orcid.org/0000-0002-7252-902 I \\ Chief Occupational Therapist, Chris Hani Baragwanath Academic Hospital, Johannesburg, South Africa.
}

\author{
Pragashnie Govender, B.OT (UKZN); M.OT (UKZN); PhD (UKZN). \\ https://orcid.org/0000-0003-3 I55-3743 \\ Associate Professor, School of Health Sciences, University of KwaZulu-Natal.
}

December Mpanza, BOT (UKZN); MOT (UKZN). http://orcid.org/0000-0003-2777-9256

Lecturer, Discipline of Occupational Therapy, School of Health Sciences, University of KwaZulu-Natal.

Background: The ICAM splint programme was developed in the 1980s and is known to produce favourable results in the management of extensor tendon repairs from zone IV to zone VII. It has only become popular in South Africa over the past several years and to the authors' knowledge its application in South Africa has not previously been reported.

Aim: The study sought to describe the use of the ICAM splint programme following an extensor tendon repair from zone IV to VII at a specialised hand unit in SA.

Methods: The study followed a concurrent mixed methods design. In phase one, a retrospective file audit was conducted with a sample of patients following the ICAM programme $(n=75)$. Phase two involved individual therapist interviews $(n=3)$, surgeon interviews $(n=2)$ and a focus group with physiotherapists and occupational therapists $(n=7)$ that had experience with the ICAM splint programme. Microsoft excel was used to descriptively analyse phase one data and thematic analysis was used to analyse phase two data. Data were merged and represented in a joint display.

Results: The majority of patients (83\%) were male with $40 \%$ in the age range of $26-35$ years old. The injuries were mainly violence related (69\%) in comparison to accidental injuries (3/\%) with extensor tendon zone V (40\%) and VI (47\%) being the most commonly involved. Adherence was reportedly influenced by factors such as patients' financial status, education and language barriers.

Conclusions: The results suggest that the ICAM splint programme is beneficial in managing extensor tendon repairs from zone IV to VII. Therapists should consider factors that affect non-adherence when selecting patients for the ICAM programme to determine its suitability. Educating patients on the ICAM splint programme is essential to facilitate adherence. Novice therapists to the ICAM programme should receive formal training to improve confidence and skill.

Key words: ICAM splint programme, extensor tendon repair, zone IV to VII

\section{INTRODUCTION}

Tendon injuries of the hand managed by occupational therapists and physiotherapists in South Africa (SA) can be difficult as they often respond poorly to treatment and therefore require prolonged rehabilitation'. Extensor tendon injuries can cause significant impairment, yet the assessment and management of these injuries have not been widely reviewed in comparison to flexor tendon injuries ${ }^{2,3,4}$. These injuries may be challenging to manage due to the complex anatomy of the extensors as any minimum discrepancies in tendon length and tension can cause significant functional deficits following injury and repair $^{5}$. In addition to the complex anatomy, there are various contextual factors within SA that makes rehabilitation of upper limb injuries different to that of developed countries ${ }^{6}$. As a result, this may influence the rehabilitation of extensor tendon repairs.

Rehabilitation programmes for extensor tendon repairs can be perplexing, making it difficult for occupational therapists striving towards excellence in clinical practice ${ }^{7}$. Post-operative rehabilitation programmes include immobilisation (IM), early passive motion (EPM) and early active motion (EAM) $)^{7,8}$ and more recently the Immediate Controlled Active Motion (ICAM) splint programme to manage extensor tendon repairs from zone IV to VII9. The ICAM splint comprises of a wrist extension splint positioning the wrist in $20^{\circ}-25^{\circ}$ extension and a yoke positioning the MCPJ's in $15^{\circ}-20^{\circ}$ of relative hyperextension ${ }^{9}$ (Figure I on page 24). This technique protects the repaired tendon by decreasing the tension on it and positioning the injured digit in relative hyperextension to the uninjured digits through the use of the yoke splint ${ }^{9}$. A study conducted in the United States of America (USA) showed that the ICAM splint produced 96 percent 'excellent' and 93 percent 'good' results according to 

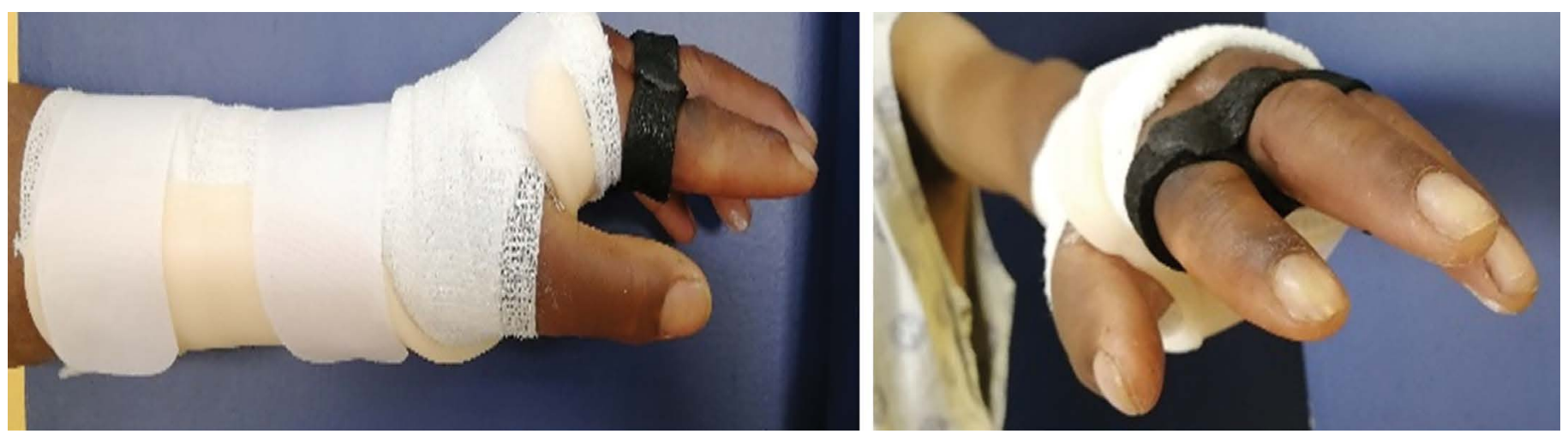

Figure I. The ICAM splint (photograph credit to first author)

Miller's criteria ${ }^{10}$ (Table I on page 25). The first edition of the ICAM splint was proposed in the early $1980 s^{9}$ but it has only recently become popular in SA.

Use of the ICAM splint programme could be valuable in managing extensor tendon repairs however, to the authors' knowledge, its application within the South African context is not well documented. The study therefore sought to describe the use of the ICAM splint programme following an extensor tendon repair from zone IV to VII at a specialised hand unit in SA.

The initial objective of the study was to describe the profile of patients that used the ICAM splint programme, followed by a description of extensor tendon function of the hand using Miller's Criteria ${ }^{10}$ based on active composite finger flexion and extension at week four, week six, week eight and week I2. The final objective of the study was to explore the experiences of the multidisciplinary team (hand surgeons, occupational therapists, physiotherapists) in implementing the ICAM splint programme.

\section{LITERATURE REVIEW}

Various rehabilitation programmes used to manage extensor tendon repairs have been developed over the years. While the focus of this study is on the use of the ICAM splint programme, an understanding of other rehabilitation programmes would assist in gaining perspective on the progression of the ICAM splint programme.

\section{Rehabilitation of Extensor Tendon Repairs from Zone IV to VII}

Due to the anatomic complexity of extensor tendons in the hand, the restoration of movement and gliding post repair is vital ". Following a repair, various factors need to be considered when deciding on which rehabilitation programme to follow. Implementing a specific programme involves consideration of the severity of injury, quality of the repair, complexity of the programme and patient adherence ${ }^{12}$. The extent and zone of the injury as well as the timing and type of repair will also determine which rehabilitation programme to select ${ }^{13}$.

Historically IM was the standard procedure following extensor tendon repairs ${ }^{9}$, however with a high risk of complications such as tendon adhesions, joint stiffness ${ }^{5}$, extensor lag and prolonged treatment $^{12}$. As a result, decreased composite flexion occurred ${ }^{14,15}$. In response, both EPM and EAM protocols have been developed to reduce complications related to the IM protocol ${ }^{13}$.

In the mid 1980s, post-operative care for repaired extensor tendons in zones IV to VII began to move from IM to controlled EPM by using a dynamic extension splint ${ }^{15}$. While EPM prevents adhesions and preserves flexion, it was found that the bulkiness of the splint interferes with daily function, that it is costly to fabricate and cumbersome to use ${ }^{15,16}$.
Controlled EAM protocols were advocated by surgeons in the 1990's as these protocols were designed to limit tendon adhesion, maintain joint mobility and are easy to fabricate ${ }^{5}$. EAM protocols encourage active mobilisation of the repaired tendon during the early post-surgical phase ${ }^{12,17}$. They however risk weakening the repaired tendon with a possibility of tendon rupture ${ }^{14}$. Patients need to be carefully selected as issues of adherence can lead to poor outcomes ${ }^{5}$. However, when successful, it helps patients regain full range of motion (ROM) earlier and with improved grip strength and quicker return to work ${ }^{14}$.

\section{Immediate Controlled Active Mobilisation (ICAM)}

In the 1980s, an advancement in the rehabilitation of extensor tendon repairs had been the Merritt relative motion splint (also known as an ICAM splint). This technique protects the repaired tendon by decreasing tension on it, positioning the injured digit in relative hyperextension to the uninjured digits through the use of a yoke splint ${ }^{13}$. The low profile of the splint makes it easy and less cumbersome to use and provides protected functional use which can increase patient adherence ${ }^{15}$.

The ICAM splint programme can be divided into three pha$\operatorname{ses}^{13}$. During phase one (0-2I days post-operative) the patient is required to wear the yoke and wrist extension splint ${ }^{9}$. In phase two (22-35 days post-operative) the patient must wear the yoke at all times however if they engage in heavy duty tasks they must wear the yoke and wrist extension splint ${ }^{9}$. In phase three (36-49 days post-operative) the wrist splint is discarded and the finger yoke is worn'. The patient must adhere to wearing the yoke constantly for the initial six-weeks ${ }^{18}$ and follow specific exercises at each phase. According to Howell and colleagues ${ }^{9}$ the average time of discharge post repair was seven weeks. In comparison to other extensor tendon programmes, the ICAM splint programme also produces favourable results and the splint design is less restrictive for patients.

\section{Assessment post-Extensor Tendon Repair}

Miller's ${ }^{10}$ guidelines (Table I, page 25) use active composite finger extension to determine the results of extensor tendon repairs?.

Total active motion (TAM) is used to describe the full arc of motion of individual digits ${ }^{19}$. This can be compared to the TAM of the contralateral digit or it can be compared to the norm of 260 degrees $^{10}$. A study conducted by Kadah ${ }^{20}$ that evaluated the results of acute extensor tendon repairs used Miller's ${ }^{10}$ classification based on TAM.

Grip and pinch strength are also accepted measures of hand function but do not directly assess extensor function?. Dynamometer measures are still however useful as it assists 
Table I. Miller's criteria for assessing extensor tendon function

\begin{tabular}{|l|l|l|}
\hline Results & $\begin{array}{l}\text { Total extensor lag } \\
\text { (degrees) }\end{array}$ & $\begin{array}{l}\text { Total flexion loss } \\
\text { (degrees) }\end{array}$ \\
\hline Excellent & 0 & 0 \\
\hline Good & $<10$ & $<20$ \\
\hline Fair & $1 \mathrm{l}-45$ & $21-45$ \\
\hline Poor & $>45$ & $>45$ \\
\hline
\end{tabular}

in determining hand function. The JAMAR dynamometer has been recommended by the American Society for Surgery of the Hand and The American Society for Hand Therapists to assess grip strength ${ }^{19}$. The test has been determined to be accurate and reliable and is recommended to compare readings with the opposite hand if not injured ${ }^{19}$.

Within the American context the ICAM splint programme has been found to produce 'excellent' to 'good' results according to Miller's criteria. While it is noted to be successfully implemented in the USA we have limited knowledge of its application within the South African context.

\section{Hand Therapy in the South African Context}

Prior to understanding the implementation of specific protocols within the South African context, it is important to understand the relevant factors with respect to hand therapy within a developing country, in order to appreciate the unique challenges that may be posed. Occupational therapists and physiotherapists in SA provide hand therapy in both the public and private sector. Health care services in the public sector are provided at different levels of care which include level one (Primary Health Care Clinic, Community Health Care Centre, District Hospital), level two (Regional Hospital), level three (Provincial Tertiary Hospital) and level four (Central Hospital, Tertiary Hospital) to meet the needs of a diverse South African population ${ }^{21}$. Within the country, there are also challenges that are common in developing contexts. These include fragmentation of health services, over-burdened and under-resourced facilities, a lack of knowledge and poor referrals as well as delays in surgical interventions in some cases ${ }^{4,6}$.

Within this milieu, a few studies to date have been valuable towards increasing our understanding of the contextual factors that influence therapy in this country ${ }^{4,6,22,23}$. De Klerk and colleagues ${ }^{22}$ highlighted tendon injuries as one of the most common injuries in SA, and were successful in highlighting assessment practices of therapists working in the field. Their study also demonstrated the limitations that were noted with respect to the assessment of activity and participation, a fundamental goal of occupational therapy. In a subsequent commentary, barriers to occupation-based hand therapy in the country were explored from the literature, experience and anecdotal evidence ${ }^{6}$. These included time, perceptions of colleagues and expectations of doctors, client follow-up, physical environment, language and knowledge ${ }^{6}$. The authors continued to advocate for more appropriate and holistic management of clients presenting with hand injuries in the country ${ }^{6}$.

Despite these studies, there was only one study that considered early active mobilisation protocols, albeit with flexor tendon injuries in the SA context ${ }^{4}$. In their feasibility study, Naude and de Klerk ${ }^{4}$, report on the functional outcomes of an early active mobilisation flexor tendon repair protocol compared to a passive mobilisation flexor tendon repair protocol in the developing context of SA. No studies have reported on early active mobilisation protocols in the management of extensor tendon repairs.

\section{METHOD}

Study Design: The study adopted a concurrent triangulation design with two phases carrying equal weight. This design allowed for use of both qualitative and quantitative data to accurately define relationships among the variables of interest ${ }^{24}$ and to broaden and strengthen the study's conclusion ${ }^{25}$. The two phases included, (i) a retrospective file audit and (ii) therapist focus group and interviews as well as interviews with surgeons.

Selection and Sampling: Census sampling was achieved for the file audit as all patient files that recorded use of the ICAM splint programme between January 2015 and December 2018 were retrieved. The setting for both phases was at a specialised hand unit at a central public hospital in SA. The hospital is located in an urban settlement in the city of Johannesburg. Patients following the ICAM splint programme - and who had received their rehabilitation by physiotherapists and occupational therapists that work in the Hand Unit - were included in the study. The physiotherapists focused on range of motion and strengthening during the ICAM splint programme while the occupational therapists focused on splinting, scar management and hand function. Patients that followed any other protocol were excluded from the study.

\section{Phase I: File Audit (Quantitative phase)}

\section{Pilot study:}

A pilot study was conducted to increase reliability and control measurement error as well as to trial the data extraction instrument. Five patient files were accessed for this purpose. The data extraction tool was compiled solely for the purpose of this study and was based on information recorded in patient files by occupational therapists, physiotherapists and surgeons. The pilot study included tick boxes in the data extraction tool with some of the variables based on common terminology used in patient files e.g. mechanism of injury, description of the wound, reasons for remoulding the yoke splint.

\section{Selection and Eligibility Criteria:}

Following the pilot study, a total of 473 patient files were identified and screened of which 75 files were included as they met the inclusion criteria (Figure 2 on page 26). Male and female patients over 18 years old were included. Patients that underwent an extensor tendon repair from zone IV to VII of one or two digits and followed the ICAM splint programme between January 2015 up to and including December 2018 were included. Patient files were excluded if there was a previous injury on the hand, the ICAM splint programme was not followed or if there was combined flexor and extensor tendon injuries on the same hand.

\section{Data Collection:}

Information from day one post-surgery until I 2 weeks post-surgery was retrieved (Table II on page 26) using the data extraction tool. Information was transferred onto an Excel sheet using Microsoft Excel 2016 for data analysis.

\section{Data Analysis:}

Data were organised onto a spreadsheet and coded using numerals for each patient for descriptive analysis. The categorical variables as 
Table II. Brief overview of data extracted from patient files

\begin{tabular}{|c|c|}
\hline & Data Extracted \\
\hline \multirow{4}{*}{ Demographic Details } & Age band \\
\hline & Gender \\
\hline & Affected hand \\
\hline & Dominance \\
\hline \multirow{8}{*}{ Injury and Surgical Details } & Date of Injury \\
\hline & Date of surgery \\
\hline & Mechanism of injury (Knife, panga, bottle glass, fence, fall, other) \\
\hline & Violence or accidental injury \\
\hline & Zone involved \\
\hline & Other co-morbid injury \\
\hline & Affected digit \\
\hline & Affected tendon (EDC, ECU, EIP, EDM) \\
\hline \multirow{4}{*}{$\begin{array}{l}\text { Day I } \\
\text { Post- Surgery }\end{array}$} & Description of the wound (clean, slough, septic, macerated, dry, healed, other) \\
\hline & Presence or absence of oedema (yes, no) \\
\hline & Did the therapist provide education to the patient on the ICAM splint programme? (yes, no) \\
\hline & If the patient reported pain or not? (yes, no) \\
\hline \multirow{7}{*}{$\begin{array}{l}\text { Week I,2,3 } \\
\text { Post-Surgery }\end{array}$} & Description of the wound (clean, slough, septic, macerated, dry, healed, other) \\
\hline & Presence or absence of oedema (yes, no) \\
\hline & Did the therapist provide education to the patient on the ICAM splint programme? (yes, no) \\
\hline & If the patient reported pain or not? (yes, no) \\
\hline & Reason for remoulding the yoke splint (tight, loose, broke, discomfort, incorrect position, lost yoke, other) \\
\hline & Adherence with splint and exercises as recorded by the therapist (yes, no) \\
\hline & Attendance to appointment or not (yes, no) \\
\hline \multirow{10}{*}{$\begin{array}{c}\text { Week } 4 \\
\text { Post-Surgery }\end{array}$} & Description of the wound (clean, slough, septic, macerated, dry, healed, other) \\
\hline & Presence or absence of oedema (yes, no) \\
\hline & Did the therapist provide education to the patient on the ICAM splint programme? (yes, no) \\
\hline & If the patient reported pain or not? (yes, no) \\
\hline & Reason for remoulding the yoke splint (tight, loose, broke, discomfort, incorrect position, lost yoke, other) \\
\hline & Adherence with splint and exercises as recorded by the therapist (yes, no) \\
\hline & Attendance to appointment or not (yes, no) \\
\hline & Goniometric AROM readings of the affected digit \\
\hline & Calculation of TAM \\
\hline & $\begin{array}{l}\text { Assessing extensor tendon function using Miller's criteria (Miller's total extensor lag classification, Miller's total flexion loss } \\
\text { classification) Rated as excellent, good, fair and poor. }\end{array}$ \\
\hline \multirow{11}{*}{$\begin{array}{l}\text { Week 6-8 } \\
\text { Post-Surgery }\end{array}$} & Description of the wound (clean, slough, septic, macerated, dry, healed, other) \\
\hline & Presence or absence of oedema (yes, no) \\
\hline & Did the therapist provide education to the patient on the ICAM splint programme? (yes, no) \\
\hline & If the patient reported pain or not? (yes, no) \\
\hline & Reason for remoulding the yoke splint (tight, loose, broke, discomfort, incorrect position, lost yoke, other) \\
\hline & Adherence with splint and exercises as recorded by the therapist (yes, no) \\
\hline & Attendance to appointment or not (yes, no) \\
\hline & Goniometric AROM readings of the affected digit \\
\hline & Calculation of TAM \\
\hline & $\begin{array}{l}\text { Assessing extensor tendon function using Miller's criteria (Miller's total extensor lag classification, Miller's total flexion loss } \\
\text { classification) Rated as excellent, good, fair and poor. }\end{array}$ \\
\hline & Dynamometer Readings \\
\hline
\end{tabular}

listed below were analysed using Microsoft Excel 2016 descriptive statistics of which the frequency and proportions were represented on bar graphs or pie charts. Due to missing information in files, the $n$ values across the weeks for the variables differed as the frequency and proportions were based on the actual number of files that the data were captured from in that week.

\section{Categorical variables:}

- The frequency and proportions of results based on the Miller's Criteria ${ }^{10}$ (excellent, good, fair, and poor) were represented on a bar graph at weeks four, six, eight and twelve post-operative.

- The frequency and proportion of patients' adherence to the ICAM splint programme This included adherence to splint wear, exer- cises and follow-up appointments as recorded by the therapist.

- The frequency and proportion of delayed repairs.

- The frequency and proportion of the hand affected, tendon and digits, zone of injury, number of digits affected, mechanism of injury, gender and age band.

- The frequency and proportion of violence-related injuries versus accidental injuries.

- The frequency and proportion of splint remoulding at follow-up appointments.

\section{Reliability and Validity:}

In ensuring reliability and threats to validity, a pilot study was conducted in order to appraise the data extraction tool and ensuring 


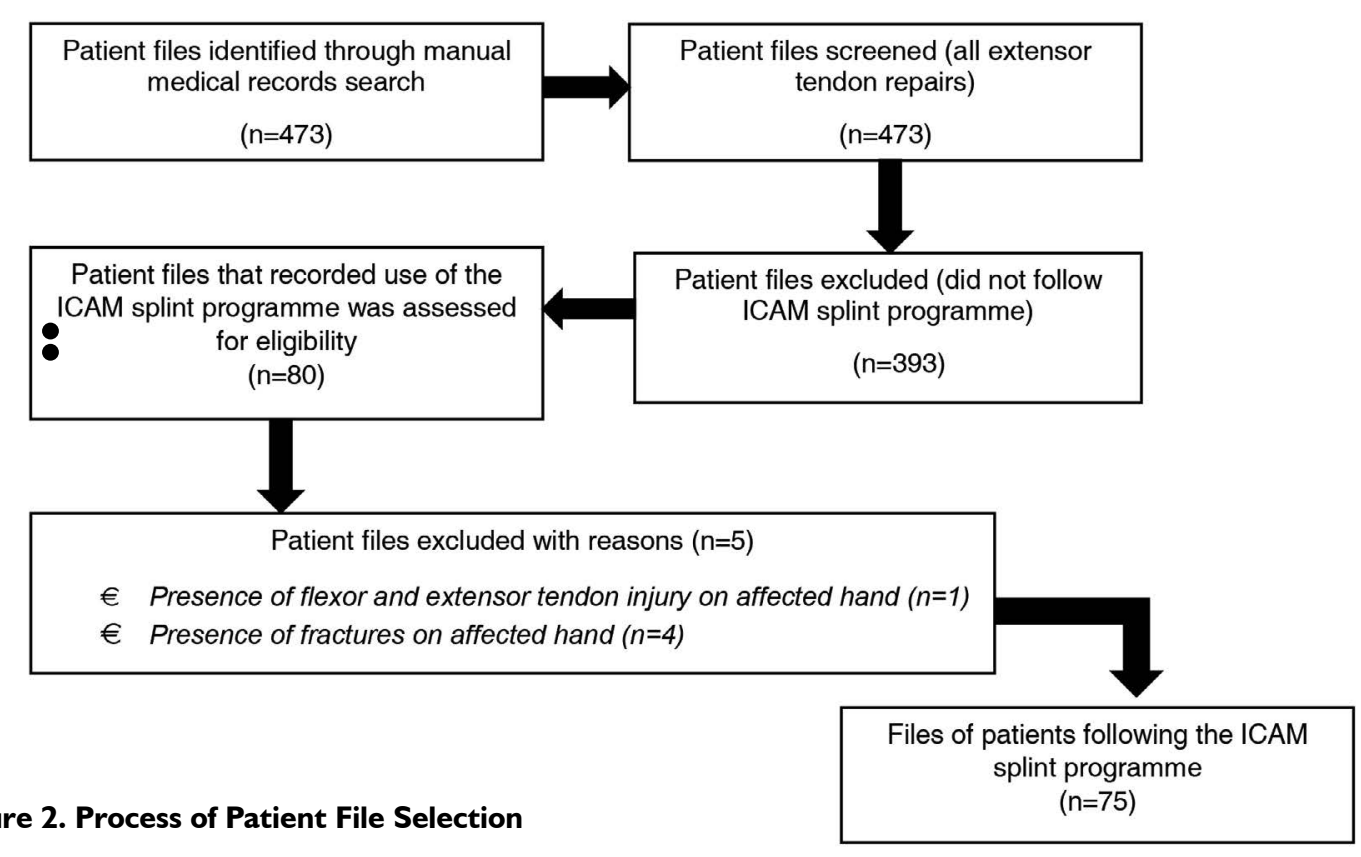

Figure 2. Process of Patient File Selection

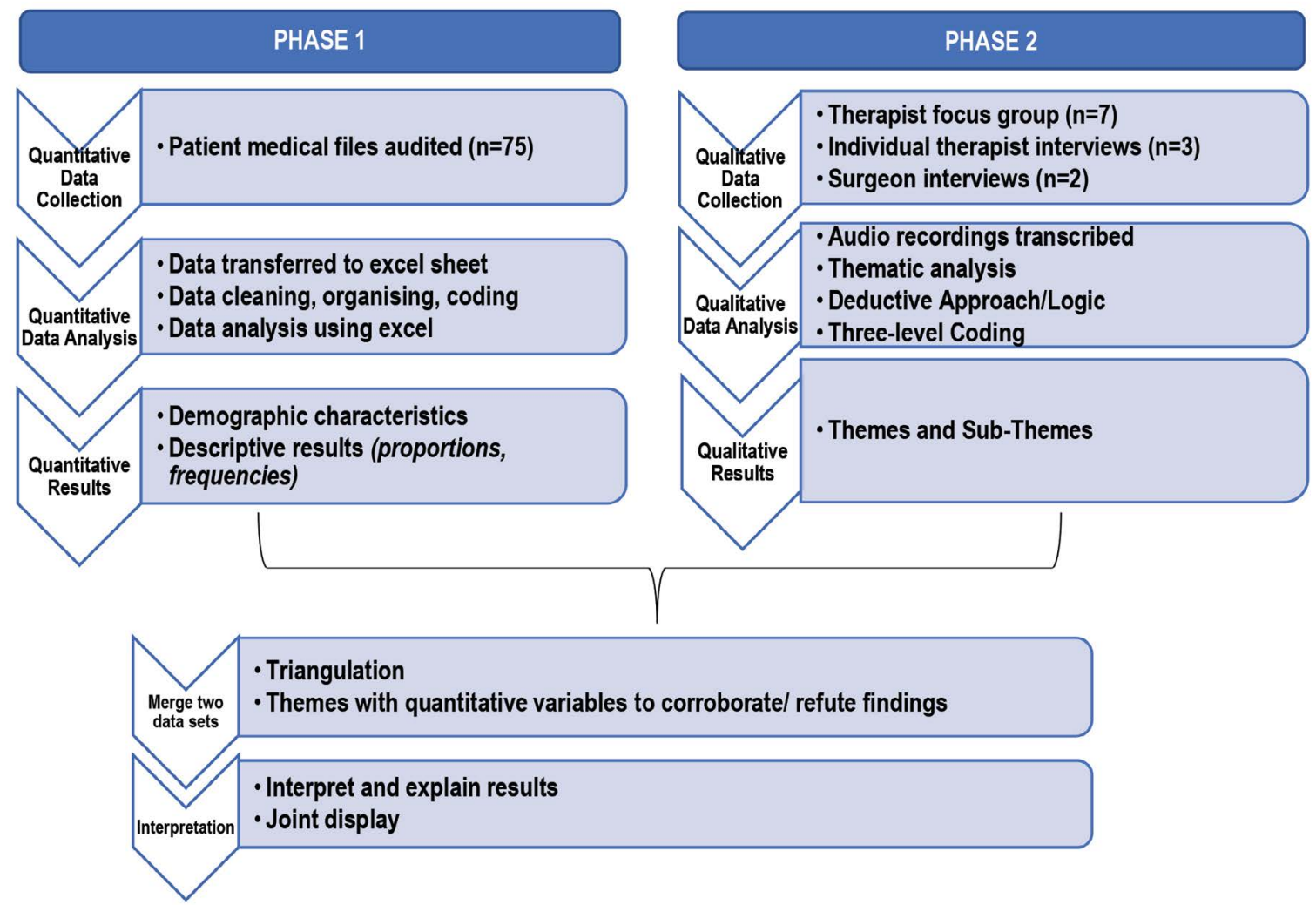

Figure 3. Overview of the Data Analysis Process

that it measured what it purported to measure. The first author was the constant in the data extraction process. This limited the variances that could have occurred in the method of data extraction.

\section{Phase 2: Interviews and Focus Groups with thera- pists and surgeons (Qualitative Phase)}

Due to the small sample size of therapists available for the focus group, further individual interviews were conducted with therapists and surgeons to supplement the information obtained from the focus groups. Therapists registered with the Health Professional Council of South Africa (HPCSA) and who have experience with the ICAM splint programme were included. Consultants and reg- istrar doctors registered with HPCSA and who have experience in extensor tendon surgery were also included.

\section{Data Collection:}

A one-hour focus group was conducted with four occupational therapists and three physiotherapists who work/had worked in the hand unit and have experience with the ICAM splint programme. A total of three semi-structured individual face-to-face interviews were conducted with occupational therapists and two surgeons from the hand unit that have experience in extensor tendon repairs. Therapists and surgeons were blinded to the results of phase $\mathrm{I}$. 


\section{Data Analysis:}

Audio-recordings of the focus group and interviews were transcribed verbatim. Thematic analysis was used for identifying, analysing, and reporting themes within the data using a deductive approach ${ }^{26}$ (see Figure 3 on page 27).

\section{Trustworthiness:}

Credibility of the study was enhanced by using purposive sampling techniques as participants with experience in the ICAM splint programme and extensor tendon repairs were recruited ${ }^{24}$. The audio-records were transcribed and findings reported using verbatim quotes. To ensure dependability, a clear exposition of data collection and data analysis methods were documented. Confirmability and dependability were achieved through a reflexive journal which allowed the identification of biases of the first author who collected the data. Data were triangulated by merging the two data sets from phase one and phase two to strengthen the study results. Transferability was achieved by providing a thick description on the study participants and the context of the study ${ }^{25}$.

\section{Merging of Data:}

A concurrent triangulation strategy was used to merge the data from the two phases. Following analysis of data from phase one and phase two, the point of integration occurred at the results stage (Figure 3 on page 27) at which the analysed qualitative and quantitative data were merged to corroborate and triangulate findings using concurrent triangulation strategy ${ }^{27}$. A joint display was used, with the quantitative findings represented on bar graphs and pie charts and the qualitative findings listed beside it organised into themes and sub-themes ${ }^{27}$. This allowed side-by-side comparison to corroborate findings. Findings were listed below the joint display in a narrative form.

\section{Ethical Clearance and Considerations:}

Ethical approval was obtained from the Biomedical Research Ethics Committee of the University of KwaZulu-Natal (BE66I//8) and gatekeeper permission received from the Medical Advisory Committee at the central public hospital. In phase one confidentiality was maintained in keeping with the Protection of Personal Information Act (PoPIA) 2018 by ensuring that all data captured from patient files were de-identified ${ }^{28}$. In phase two informed consent was obtained from all participants and aliases used when transcribing the data.

\section{RESULTS}

\section{Demographic Profile of Patients following the ICAM Splint Programme $(n=75)$}

Patients following the ICAM splint programme comprised $83 \%$ $(n=62)$ male and $17 \%(n=13)$ female. Forty percent $(n=30)$ of patients were between the age range of 26-35 years old, followed by $27 \%(n=20)$ between $18-25$ years old, $23 \%(n=17)$ between $36-45$ years old, $9 \%(n=7)$ between $46-55$ years and $1 \%(n=1)$ above 56 years old. Only unilateral injuries were noted, of which $53 \%(n=40)$ of patients had right hand involvement and $47 \%(n=35)$ with left hand involvement. Of these, $57 \%(n=28)$ of patients had their dominant hand affected.

\section{Demographic Profile of Therapists and Surgeons $(n=12)$}

Therapists in phase two $(n=10)$ comprised of $30 \%(n=3)$ physiotherapists and $70 \%(n=7)$ occupational therapists. The mean age of therapists was 29 years old with majority of them $(70 \% ; n=7)$ with less than two years' experience in hand therapy. All therapists reported treating less than five patients with the ICAM splint programme per month. The physiotherapists $(30 \% ; n=3)$ reported receiving no formal training on the programme and the occupational therapists (70\%; $n=7)$ reported learning about the programme through peer learning and observation. The mean age of the surgeons $(n=2)$ was 35 years old. All participants worked in the public sector.

\section{Details of the Injury}

Of the total patients profiled, $69 \%(n=49)$ sustained their injury due to violence-related incidents and $31 \%(n=22)$ were associated with accidental injury. The mechanism of injury recorded in files $(n=68)$, revealed $35 \%(n=24)$ was due to stab injuries with a knife, $1 \%(n=I)$ with a panga, $21 \%(n=14)$ with a bottle, $15 \%(n=10)$ with glass, $1 \%(n=1)$ falling on a fence, $6 \%(n=4)$ from a fall and $21 \%(n=14)$ was unclassified (Figure 8 on page 31$)$. In terms of digits involved, $63 \%(n=47)$ of patients had one digit involved and $37 \%(n=28)$ had two digits. The extensor tendons injured included extensor digitorum communis $(71 \% ; n=68)$ ), extensor indicis proprius (I5\%; $n=\mid 4)$ ), extensor digiti minimi $(I \mid \% ; n=I I))$ and extensor carpi ulnaris (3\%; $n=3)$.

\section{Patient Adherence with the ICAM Splint Programme Improved Adherence}

As reported by therapists, patients were more inclined to follow the ICAM splint programme in terms of splint adherence, exercise adherence and attending follow-up appointments in relation to the immobilisation programme. This information corroborated with the file audit as illustrated in Figure 3 on page 27.

...they are pretty compliant even though they might not come on their day, they walk in and say I couldn't pay to come this day but I am here today. (Focus group-physiotherapist)

Therapists proposed that patients were more adherent with the ICAM programme in comparison to the immobilisation programme because they could monitor and track their progress. The patients' perception of improvement motivated them to comply with exercises.

It also motivates the patient because they can see more progress, how much they can flex and extend each week whereas in the extended wrist extension they only have PIP flexion and they can't see the hand actually moving. (Focus group-Occupational Therapist)

\section{Factors affecting Non-Adherence}

Adherence with the splint and the exercise programme was higher in comparison to follow-up appointments. The highest rate of appointments not attended was at week $4(40 \%)$, week $6(47 \%)$ and week $8(55 \%)$. One of the influencers mentioned by the participants was limited finances and the patient's perception of the hand being fully functional.

The patients will tell you that they don't have money to come to hospital for an extensive rehabilitation programme... (Surgeon interview-Registrar)

... they might just think that my hand is working and I don't need to return to the hospital so then they (are) not necessarily defaulting, the hand is fully functional, they are just not coming back to pay for another appointment. (Focus group-Occupational Therapist) 
The level of education of the patient and their insight into the importance of rehabilitation were identified as contributors to adherence. Therapists and surgeons alike expressed the responsibility of the MDT to educate the patient at every session on the importance of adhering to the ICAM programme. Therapist recordings of patient education in the hospital file was highest on day one post-operative $(76 \% ; n=57)$ and decreased by week $12(31 \% ; n=23)$.

...it is very dependent on understanding, the patient's compliance and also how we stress the importance of it as well to try and educate as much as possible every single session... (Focus group-Occupational Therapist)

Surgeons also identified the importance of education about the surgical procedure to promote adherence with therapy post-surgery.

...informing the patient is important...you can't blame the patients if they haven't been explained (to) by the surgeons and therapists... (Surgeon interview-registrar)

Language and comprehension was recognised as a barrier to adherence. Therapists expressed that although the ICAM pro- gramme is explained to patients there can be miscommunication due to a language barrier.

... a language barrier where they don't understand what you are saying and sometimes that affects their compliance. (Focus group-Physiotherapist)

According to therapists, patients easily removed the finger yoke due to the splint design. Non-adherence with the finger yoke was highest after discontinuation with the wrist extension splint.

...because they can remove the finger yoke and sometimes it isn't completely immobilised like you would want... (Focus groupOccupational Therapist)

Therapists' reasons for their preference of the ICAM splint programme

\section{Improved ROM and Mobilisation}

Therapists shared their experiences with the immobilisation programme in comparison to the ICAM programme. The ICAM programme was favoured as therapists expressed that patients pre-

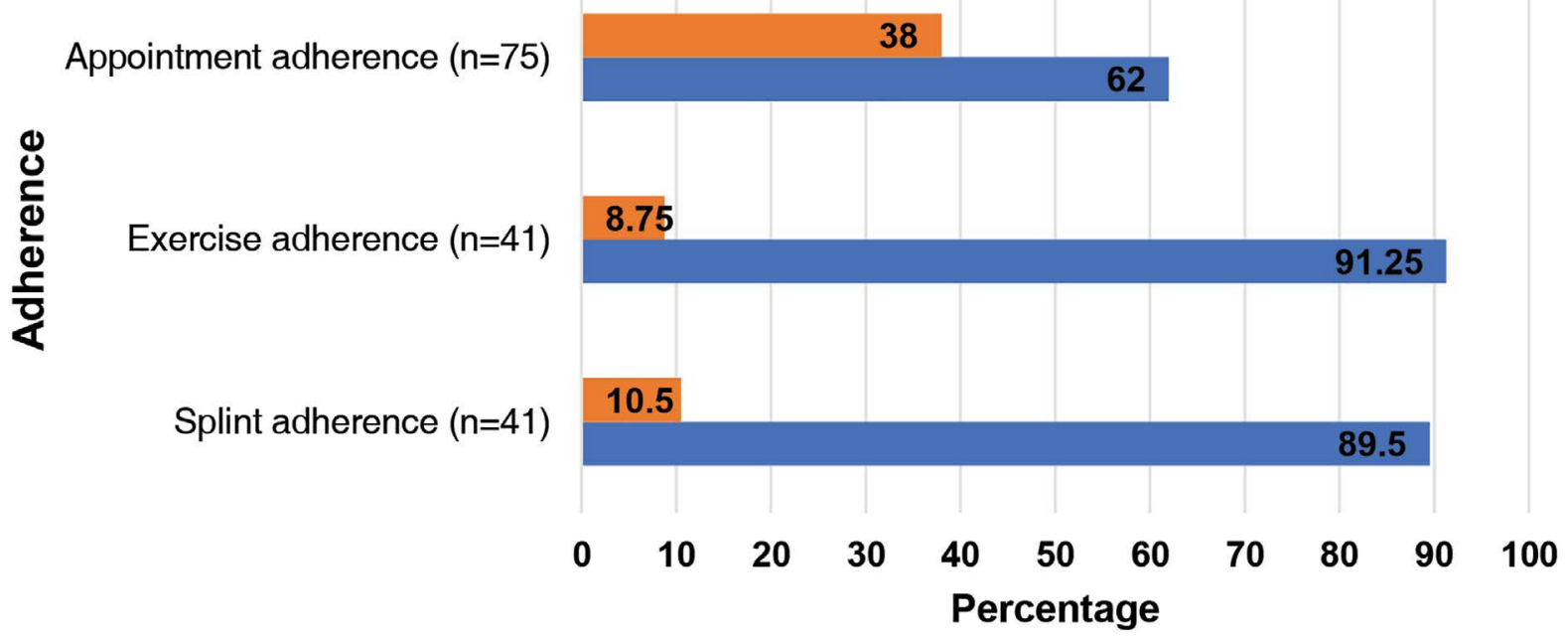

Figure 4. Patient Adherence with the ICAM Splint Programme

25

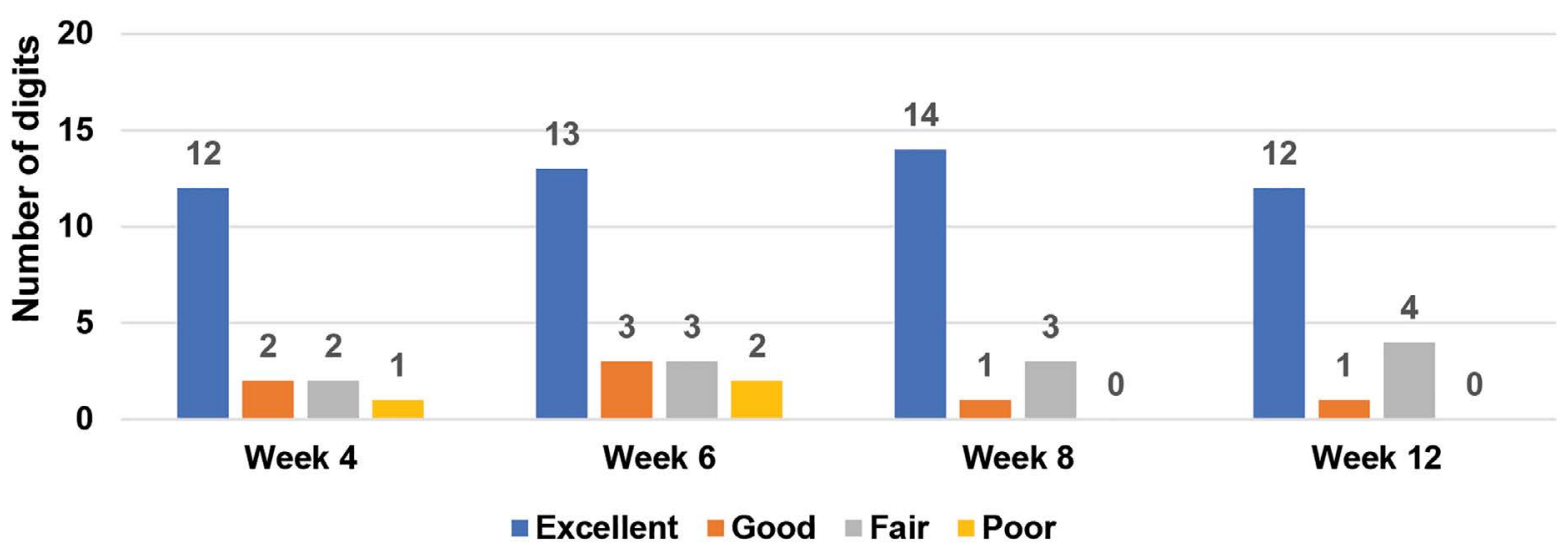

Figure 5. Extensor lag classification ${ }^{10}$ at each week 


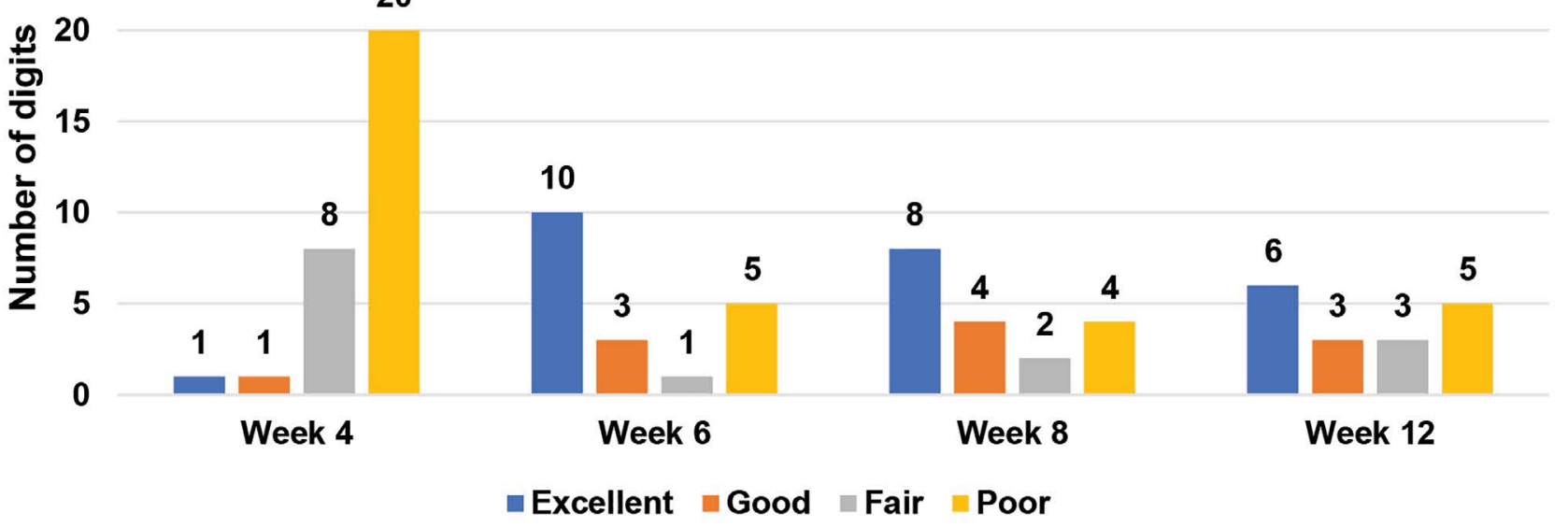

Figure 6. Flexion Loss Classification ${ }^{10}$ at each week

sented with better ROM at the metacarpal phalangeal joints (MPJ), shorter therapy time and fewer tendon adhesions. The physiotherapists agreed that it was easier to mobilise the patient at four weeks, as there was less stiffness compared to patients who were immobilised.

We find there is less stiffness in the MCP's than in the extended wrist extension splint because they can mobilise a lot sooner. (Individual therapist interview-Occupational Therapist)

...getting that tendon glide early on, you notice that it is a lot more successful to prevent tendon adhesions. (Focus groupOccupational Therapist)

...it is easier for us to rehab them sooner because they are already used to moving their hand but as soon as you take an immobilisation splint off they haven't moved in four weeks. (Focus group-Physiotherapist)

According to Miller's total extensor lag classification, at week 12 patients' presented with $71 \%(n=12)$ excellent, $6 \%(n=1)$ good and $0 \%$ poor results (Figure 4 on page...). At week 12 , according to Miller's total flexion loss classification patients' presented with $29 \%$ $(n=4)$ excellent and $35 \%(n=6)$ poor results (Figure 5 on page 29).

\section{Earlier return to Work}

Therapists reported that the ICAM programme allowed patients to engage in light duty activities of daily living while in the splint and in some instances, an earlier return to work.

...the fingers are free so you can do lighter duty type of things... admin duties so they could either be put in a lighter role or light duty at work... (Focus group-Occupational Therapist)

\section{BARRIERS TO IMPLEMENTING THE ICAM SPLINT PROGRAMME}

Training and knowledge of ICAM splint programme Therapists identified training on the ICAM splint programme as being insufficient. Occupational therapists attended peer-learning events or learnt the programme through observation. The physiotherapists reported receiving no formal training. Therapists expressed that their confidence using the programme improved over time. Newly qualified therapists were considered to experience more difficulties in implementing the programme. Due to regular rotation of physiotherapists within the unit, they expressed concerns that therapists had to be frequently orientated to the ICAM programme and the issue of limited knowledge.

...now I feel confident for the amount of time that I had to practice but initially I wasn't as confident. (Focus group-Occupational Therapist)

... as physio's (physiotherapists) sometimes we get confused because we rotate often...we always like need constant reminding what the process is and then you have to remind different people... (Focus group-Physiotherapist)

\section{Fabricating the Finger Yoke}

Occupational therapists recognised difficulties with fabricating the finger yoke. Achieving the correct degree of relative hyperextension to the uninjured digit was challenging. Ease of fabrication was dependent on which digits were involved. The middle finger was most commonly involved with (33\%; $n=34)$, index finger $(31 \% ; n=32)$, ring finger $(19 \% ; n=20)$ and little finger $(17 \% ; n=17)$. Therapists experienced difficulty correctly positioning an affected little finger in the yoke in comparison to other digits. Remoulding of the finger yoke on follow-up visits was common but this decreased over time. As recorded in the patients' files, the top three reasons for remoulding was discomfort (42\%; $n=19)$, the yoke fitting too tightly $(18 \%$; $n=4)$ and splinting in the incorrect position ( $13 \% ; n=3)$. Therapists expressed that difficulties with yoke fabrication and remoulding (see Figure 7 on page $3 \mathrm{I}$ ) was further influenced by oedema and patients' pain threshold.

\section{Objective Testing}

Physiotherapists reported using the dynamometer to assess grip strength and the goniometer to assess ROM to monitor progress of the patient. The occupational therapists reported using clinical observation and recognised the need to use more objective assessments to better monitor progress and functional outcomes.

\section{Surgeon perspectives on factors influencing outcomes in Extensor Tendon Repairs}

A number of factors were considered to influence the outcomes of extensor tendon repairs and included the mechanism of injury, delayed repairs, zone of injury, wound healing and infection. 


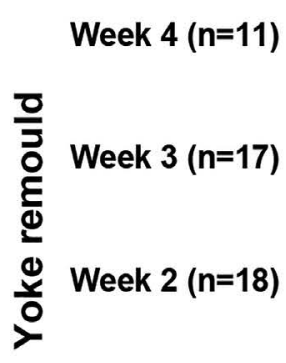

Week $1(n=26)$ 0

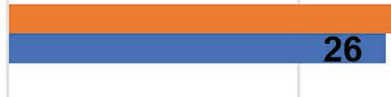
26

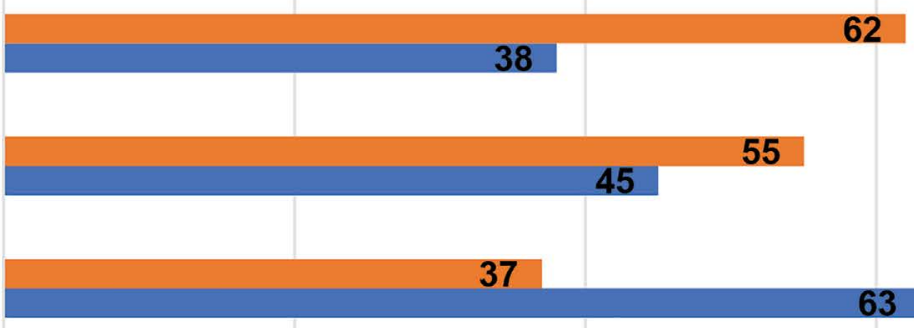

20

40

Percentage

Yoke not remoulded $\quad$ Yoke remoulded

Figure 7. Remoulding of the Finger Yoke at weeks I $(n=26)$, week $2(n=I 8)$, week $3(n=I 7)$ and week $4(n=I I)$

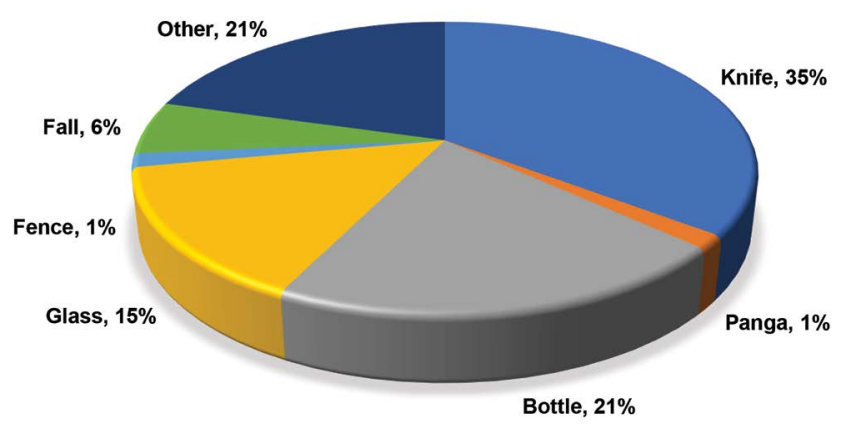

Figure 8. Mechanism of Injury $(n=68)$

Surgeons reported that the mechanism of injury can influence the surgical repair. Knife injuries was the highest at 35\% $(n=24)$ (Figure 8 on page 31 ). Injuries with sharp objects made surgery easier in comparison to bottle injuries $(21 \% ; n=14)$ whereby patients presents with multiple jagged cuts. The majority of extensor tendon injuries treated by the surgeons was related to interpersonal violence, which they reported as easier to manage because the weapon was often sharp resulting in less complicated cuts.

Bottle injuries are the ones where we struggle because there are multiple cutting surfaces you get jagged edges which makes their soft tissue compromised... (Surgeon interview-registrar)

Delayed repairs, due to environmental barriers, was also identified as a challenge in managing extensor tendon repairs. Surgeons reported that this was attributed to large patient loads resulting in delays in theatre availability, patients arriving late and delayed referrals from satellite hospitals.

From the file audit $(n=74)$ it was identified that $26 \%(n=19)$ of patients underwent surgery between day 0-2 of the injury, $51 \%(n=38)$ between day 3-7, $23 \%(n=17)$ between day $8-14$ and $0 \%$ above 14 days.

One of the surgeons recognised the zone of injury as a factor that influenced surgical results. From the file audit the majority of the injuries occurred over zone $\mathrm{V}(40 \% ; \mathrm{n}=24)$ and zone $\mathrm{VI}$ (47\%; $n=28)$ and I $3 \%(n=8)$ over zone VII. Surgeons considered the anatomy of the different zones as a factor that affected outcomes post-repair.
...I think zone IV, V, VI are okay once you start going towards zone VII and muscle belly and the muscular tendinous junction then it becomes tricky... (Surgeon interview- registrar)

Surgeons acknowledged wound healing as a determinant that affected extensor tendon repairs. Acutely infected wounds post repair sometimes requires a debridement which risks rupturing the repair. Patients may then require tendon reconstruction or a tendon transfer at a later stage. Furthermore, surgeons noted that infection causes scar tissue formation often resulting in patients requiring a tenolysis. From the file audit at week 4, therapists described $47.5 \%(n=19)$ of the wounds as healed, $27.5 \%(n=I I)$ clean, $20 \%(n=8)$ dry, $2.5 \%(n=1)$ septic, $2.5 \%(n=1)$ macerated and $0 \%$ with slough. There was record of only one patient requiring debridement due to infection.

...we need to debride the tendon and end up losing our repair... (Surgeon interview-registrar)

...infection causes scarification around the area and it also causes adhesions which forces the surgeons...going back to theatre excising the scar and tenolyse the tendons... (Surgeon interview-registrar)

\section{DISCUSSION AND IMPLICATIONS}

The use of the ICAM splint programme for early active mobilisation is not well documented in South Africa. This study, which may be the first to the authors' knowledge, aimed to profile and describe the intervention of the ICAM programme at a specialised hand unit in South Africa. From this study, adherence to therapy emerged as a dominant theme. Adherence with exercises and use of the splint improved as patients progressed through therapy however, appointment follow-ups were identified as an area of concern. Defaulting of appointments were attributed to limited finances and a sense of having a fully functional hand and following removal of the wrist extension splint. This finding is in keeping with Howell and colleagues study on the ICAM splint programme, where patients were non-adherent to therapy due to insurance issues, self-discharge when doing well, within ten days or after discontinuation of the wrist extension splint. Therefore, when following the ICAM splint programme it is recommended that patients are followed-up at least once a week to check the fit of the splint, progress on the programme and to individualise 
instructions ${ }^{9}$. Non-attendance at follow-up appointments may affect the results post rehabilitation, as the programme may then not be closely monitored. This was confirmed in a South African study, in which Naude and de Klerk ${ }^{4}$ examined the feasibility criteria in a randomised pilot trial comparing two post-operative therapies for patients with flexor tendon injuries to the hand (one of which included an early active mobilisation programme). Various barriers, including recruitment, retention and use of selected outcome measures, limited the potential of the study and their trial demonstrated a failure to meet the criteria for feasibility due to contextual barriers that threatened the feasibility of such a study ${ }^{4}$.

Patient education provided by the MDT helps improve insight into the condition and the effects of non-adherence and education at each follow-up visit reinforces patient understanding. Patients' ability to recall medical information may be poor but deeper insight into the risks and consequences are said to improve knowledge ${ }^{8}$. Patient education from the MDT was deemed essential when following the ICAM programme. For EAM programmes to be successful, patients need to have good insight into the programme and into the consequences of non-adherence. Patient education can however be challenging in the South African context. South Africa is a diverse country with II official languages. Although therapists try to educate patients on the importance of therapy, language barriers result in poor understanding influencing adherence with therapy. This is supported by other South African literature ${ }^{4,6,22}$. The MDT needs to develop novel ways in which to educate patients on the surgical procedure and effects of non-adherence with the ICAM programme. Improved understanding with the use of a translator may influence patient adherence resulting in better outcomes.

Therapists favoured the ICAM programme over other extensor tendon programmes due to their experience of achieving better ROM, shorter therapy time and the functionality of the splint. According to Miller's ${ }^{10}$ criteria the majority of patients had excellent extension and poor total flexion loss by week 12 . While extensor tendon function was favourable, flexion may have been limited due to stiff joints or tendon adhesions. The limitation of flexion after an extensor tendon repair is a prominent difficulty with patients losing more flexion than extension ${ }^{2,29}$. However, in a systematic review by $\mathrm{Ng}$ and colleagues ${ }^{12}$ who considered five studies, patients following EAM extensor tendon protocols still gained significantly better TAM than those following immobilisation protocols.

The ICAM splint design allows patients to engage in basic activities of daily living and perform light duty work'. While the ICAM programme allows more freedom to use the hand in comparison to other programmes, it was noted by therapists that patients were more likely to remove the yoke due to ease of removal. In a systematic review by Wong and colleagues ${ }^{8}$, it was found that a focus on improvement in patient adherence to EAM splints and rehabilitation protocols are essential. Reasons for splint non-adherence may be attributed to poor appearance, discomfort, interference with function, failure to decrease pain, and hygiene ${ }^{8}$. Therapists reported initial challenges in fabricating the yoke and the main reason for remoulding was due to discomfort. Formal training and in-services are required to improve therapist confidence when implementing the ICAM programme. This could assist novice therapists with limited experience in splinting in ensuring that comfort and aesthetics are valued to assist with splint adherence.

As noted from the focus group physiotherapists used assessments such as ROM and grip strength while occupational therapists used observation to monitor progress. Occupational therapists recogni- sed the need to use validated assessments to determine results of using ICAM programme and functional outcomes. In their review, $\mathrm{Ng}$ and colleagues ${ }^{12}$ found that ROM and grip strength were used as measures but none of the included studies used validated patient reported outcome measures e.g. The Disabilities of the Arm, Shoulder and Hand Questionnaire (DASH) ${ }^{30}$. This was reported in the South African context by de Klerk and colleagues ${ }^{22}$ who found that therapists lacked assessment with use of measures that were responsive to activity and participation domains of the International Classification of Functioning, Disability and Health (ICF). Wong and colleagues ${ }^{8}$ reiterated that inconsistencies in how outcomes are measured, makes comparisons across studies difficult, and suggested that studies investigating tendon rehabilitation should also follow the ICF to describe functioning, disability and health in a standardised way.

External factors such as mechanism of injury, age, occupation and handedness can determine the management of extensor tendon injuries ${ }^{2}$. Although patients may be adherent to the ICAM splint programme and the implementation of the programme is followed other external factors scan still influence the outcomes. In this study the majority of injuries that occurred were violence-related and the weapon most commonly used was a knife. Based on the information gained from the surgeons in this study, these are easier to manage due to straight cuts with a sharp object. According to Griffin and colleagues ${ }^{2}$ it is important for surgeons to get background history on the mechanism of injury to determine if the wound needs debridement, if there is risk of infection or the presence of foreign bodies. Infection can delay a primary repair if the patient requires debridement and a debridement post tendon repair can risk rupturing the tendon.

Surgeons in this study reported that delayed extensor tendon repairs are not ideal however, it is not always within the surgeons' control. The majority of surgical repairs were conducted within a week. From the literature, a delay between injury and repair should not exceed seven days ${ }^{29}$, before the tendon retracts or softens, as late hand referrals for extensor tendon repairs present with complications such as joint stiffness, oedema and tendon adhesions ${ }^{7}$. Apart from delays in surgery, delays in implementing the ICAM splint programme can affect the results as there is an increased likelihood of adhesions and joint stiffness. For this reason, the authors of the ICAM programme suggested that the ICAM splint should be initiated within I 0 days of the repair. However, better results are achieved if it is started immediately or within three days post-surgery?.

Anatomical structures over the different extensor zones can affect a surgical repair and possibly affect the outcomes of the ICAM splint programme. In this study the majority of the injuries occurred over zone $\mathrm{V}$ and zone Vl. Hirth and colleagues 16 found that extensor tendon injuries are common in zones $\mathrm{V}$ and $\mathrm{VI}$ due to the superficial location and minimal soft tissue protection on the dorsum of the hand. Extensor tendon repairs over zone VII lead to greater unsatisfactory results if there is retinaculum involvement as it can result in bowstringing while zone $\mathrm{V}$ produced favourably outcomes ${ }^{\prime \prime}$. Zone $\mathrm{VI}$ repairs can result in poor results due to iatrogenic factors where suture techniques shorten the tendon by 6 $\mathrm{mm}$ which can cause an 18 degree loss of motion at the MCP and PIP joint ${ }^{29}$. Further studies into the zone of injury and its effects on therapy outcomes may be beneficial in guiding therapists during rehabilitation.

\section{LIMITATIONS}

Information gathered from the file audit was restricted to what therapists recorded and excluded outcome measures as part of their routine assessments. This has been documented to be a limitation 
in other studies ${ }^{23}$. The attrition at each follow-up appointment was also a limitation as these results were then omitted from the pooled presentation of findings. The inexperience of therapists included in this study on use of the ICAM programme as well as the small sample sizes (although census sampling was employed) were also limiting factors. Having individual interviews with the surgeons may have been restrictive in that a deeper insight on the factors that influence surgery may have been possible by collective sharing that could have been fostered, for example by a dyad interview.

\section{CONCLUSION}

The results of this mixed methods study suggests that the ICAM splint programme can be used to manage extensor tendon repairs from zone IV to VII within the SA context, however factors that affect nonadherence should be considered when selecting patients. This thus poses the challenge of whether ICAM is feasible in the current context, although this is true for any protocols used in the management of tendon injuries. Therefore, whilst the ICAM splint has shown to produce favourable results in other countries, there are contextual factors in South Africa that need to be considered. Patients' financial status, level of education and language should be considered in the decision as to whether the ICAM programme will be used with a patient as this may inevitably impact adherence. An MDT approach is recommended when educating patients on the ICAM splint programme to reiterate adherence and to ensure patient-centred care. Further training and education for novice therapists is required to build therapist confidence in application of the ICAM programme and the splint fabrication. More studies on the efficacy of the ICAM programme in the South African context are required and objective and standardised assessments should be used to monitor progress of patients using the programme.

\section{ACKNOWLEDGEMENTS}

The first author would like to acknowledge the University of KwaZuluNatal for tuition remission towards a Master's degree, the findings of which are reported in this paper, the participants in this study as well as the reviewers for their critique of the paper through the various reviews.

\section{REFERENCES}

I. Docheva D, Müller SA, Majewski M, Evans $\mathrm{CH}$. Biologics for tendon repair. Advanced Drug Delivery Reviews. 2015 Apr; 84: 222 - 39. http://dx.doi.org/10.1016/j.addr.2014.1 I.015

2. Griffin M, Hindocha S, Jordan D, Saleh M, Khan W. Management of Extensor Tendon Injuries. The Open Orthopaedics Journal [Internet]. 2012 Feb 23; 6(I): 36 - 42. http://dx.doi.org/10.2174/1874325001206010036

3. Chinchalkar S, Pipicelli J. Complications of extensor tendon repairs at the extensor retinaculum. Journal of Hand and Microsurgery [Internet]. 2016 Sep 5; 02(0I): 3 - I2. http://dx.doi.org/10.1007/s/2593-010-0008-5

4. Naudé $A B$, de Klerk $S$. Introducing early active mobilisation following flexor tendon repair in the context of care in a developing country: A randomised feasibility study. South African Journal of Occupational Therapy. 2019; 49(2): 48 - 56. http://dx.doi.org/10.17159/23103833/2019/vol49n2a8

5. Abbas AR. Evaluation of Early Active Mobilization Protocol of Extensor Tendon Repair at Zone V, VI and VII. Iraqi Academic Scientific Journal. 20I2; II(2): 211 - 9.

6. De Klerk S, Badenhorst E, Buttle A, Mohammed F, Oberem J. Occupation-based hand therapy in South Africa: challenges and opportunities. South African Journal of Occupational Therapy. 2016; 46(3): 10 - 15. http://dx.doi.org/10.17159/2310-3833/2016/v46n3a3
7. Hall B, Lee H, Page R, Rosenwax L, Lee AH. Comparing Three Postoperative Treatment Protocols for Extensor Tendon Repair in Zones $\mathrm{V}$ and $\mathrm{VI}$ of the Hand. American Journal of Occupational Therapy. 20I0 Sep I; 64(5): 682 - 8. http://dx.doi.org/ I0.50 I4/ajot.2010.0909I

8. Wong AL, Wilson M, Girnary S, Nojoomi M, Acharya S, Paul SM. The optimal orthosis and motion protocol for extensor tendon injury in zones IV-VIII: A systematic review. Journal of Hand Therapy [Internet]. 2017 Oct; 30(4): 447 - 56. http://dx.doi.org/10.1016/j.jht.2017.02.013

9. Howell JW, Merritt WH, Robinson SJ. Immediate Controlled Active Motion Following Zone 4-7 Extensor Tendon Repair. Journal of Hand Therapy. 2005 Apr; 18(2):182 - 90. http://dx.doi.org/10.1 197/j.jht.2005.02.01 I

10. Miller H. Repair of severed tendons of hand and wrist: Statistical analysis of 300 cases. Surgery, Gynecology and Obstetrics. 1942; 75: 693 - 8.

II. Mehdinasab SA, Pipelzadeh MR, Sarrafan N. Results of Primary Extensor Tendon Repair of the Hand With Respect to the Zone of Injury. Archives of Trauma Research. 2012 Oct I4; I(3): I3I - 4. http://dx.doi.org// 0.5812/atr.7859

12. Ng C, Chalmer J, Macdonald D, Mehta S, Nuttall D, Watts A. Rehabilitation Regimens Following Surgical Repair of Extensor Tendon Injuries of the Hand - A Systematic Review of Controlled Trials. Journal of Hand and Microsurgery. 2016 Sep 5; 04(02): 65 - 73. http://dx.doi.org/10.1007/s /2593-012-0075-x

13. Saunders RJ, Astifidis RP, Burke SL, Higgins JP, McClinton MA. Preface. Hand and Upper Extremity Rehabilitation. 2016. http://dx.doi.org/10.1016/b978-I-4557-5647-6.09986-x

14. Patil RK, Koul AR. Early active mobilisation versus immobilisation after extrinsic extensor tendon repair: A prospective randomised trial. Indian Journal of Plastic Surgery. 2012 Jan; 45(01): 029 - 37. http://dx.doi.org// 0.4103/0970-0358.96576

15. Burns MC, Derby B, Neumeister MW. Wyndell Merritt Immediate Controlled Active Motion (ICAM) Protocol following Extensor Tendon Repairs in Zone IV-VII: Review of Literature, Orthosis Design, and Case Study - A Multimedia Article. HAND. 2013 Jan 9; 8(I): 17 - 22. http://dx.doi.org/10.1007/s I I552-012-9488-z

16. Hirth MJ, Bennett K, Mah E, Farrow HC, Cavallo AV, Ritz M, et al. Early return to work and improved range of motion with modified relative motion splinting: a retrospective comparison with immobilization splinting for zones $\mathrm{V}$ and $\mathrm{VI}$ extensor tendon repairs. Hand Therapy. 201I Dec [cited 2019 Apr 24]; 16(4): 86 - 94. http://journals.sagepub.com/doi/l0.1258/ht.201 I.01 I0I2

17. Talsma E, de Haart M, Beelen A, Nollet F. The Effect of Mobilization on Repaired Extensor Tendon Injuries of the Hand: A Systematic Review. Archives of Physical Medicine and Rehabilitation. 2008; 89(12): 2366 - 72. http://dx.doi.org/10.1016/j.apmr.2008.06.019

18. Merritt WH. Relative motion splint: Active motion after extensor tendon injury and repair. Journal of Hand Surgery. 20 I4; 39(6): I I 87 - 94. http://dx.doi.org/10.1016/j.jhsa.2014.03.015

19. Cooper C. Fundamentals of Clinical Reasoning: Hand Therapy Concepts and Treatment Techniques. Fundamentals of Hand Therapy. Elsevier; 2007; 3 - 21. http://dx.doi.org/10.1016/b0-32-303386-5/50004-7

20. Kadah M. Evaluation of the results of management of acute extensor tendon injuries of the hand. Menoufia Medical Journal. 2015; 28(I): 149. http://dx.doi.org// 0.4103/I I 10-2098. 155973

21. Referral System: Levels of Health Care; 2014. http://www.kznhealth.gov.za/Referral-system.htm

22. De Klerk S, Buchanan H, Pretorius B. Occupational therapy hand assessment practices: Cause for concern? South African Journal of Occupational Therapy. 20I5; 45(2): 43 - 50. http://dx.doi.org/10.17I59/23।0-3833/20I5/v45n2a7 
23. Pilling T, Govender P. Profile and management of the firework-injured hand. South African Family Practice. 2016 Jan 20; 58(2): 48 - 53. http://dx.doi.org/10.1080/20786190.2015.1125167

24. Elo $S$, Kääriäinen $M$, Kanste $O$, Pölkki T, Utriainen $K$, Kyngäs H. Qualitative Content Analysis. SAGE Open. 2014 Jan 7; 4(I): 2I582440 I452263. http://dx.doi.org/I0.1 I77/2I 582440I4522633

25. Kornbluh M. Combatting Challenges to Establishing Trustworthiness in Qualitative Research. Qualitative Research in Psychology. 2015 Mar 16; 12(4): 397 - 414. http://dx.doi.org/10.1080/14780887.2015.1021941

26. Braun V, Clarke V. Using thematic analysis in psychology. Qualitative Research in Psychology. 2006 Jan; 3(2): 77 - 101. http://www.tandfonline.com/doi/abs/10.1 I91/1478088706qp063oa

27. Fetters MD, Curry LA, Creswell JW. Achieving Integration in Mixed Methods Designs-Principles and Practices. Health Services Research. 2013 Oct 23; 48(6pt2): 2134 - 56. http://dx.doi.org/10.1 I I I/1475-6773.12117

28. Guidelines L. Protection of Personal Information for South African Law Firms Protection of Personal Information Guideline; 20 I8. https:// www.golegal.co.za/protection-personal-information-guidelines/

29. Newport ML, Havlik RJ. Extensor Tendon Injuries. Plastic Surgery Secrets Plus. 2010; 820 - 4. http://dx.doi.org/10.1016/b978-0-323-03470-8.00I26-5

30. Hudak P, Amadio PC, Bombardier C, and the Upper Extremity Collaborative Group. Development of an Upper Extremity Outcome Measure: The DASH (Disabilities of the Arm, Shoulder, and Hand). American Journal of Industrial Medicine. 1996; 29: 602 - 608.

\section{AUTHOR CONTRIBUTIONS}

The first author Nicole Mottay was a student registered for the degree of Masters in Occupational Therapy in the School of Health Sciences at the University of KwaZulu-Natal. The second Pragashnie Govender and third author December Mpanza were supervisors of the student. Therefore, the contribution for the manuscript has been shared within this relationship, the conceptualisation and writing up was completed by the first author and the other authors contributed by assisting in the conceptualisation of the study and in the critical review and preparation of the paper for submission to the journal. Finalisation of the manuscript was done collaboratively.

\section{Corresponding Author}

\section{*Nicole Mottay}

Email: nicoledmottay@gmail.com 